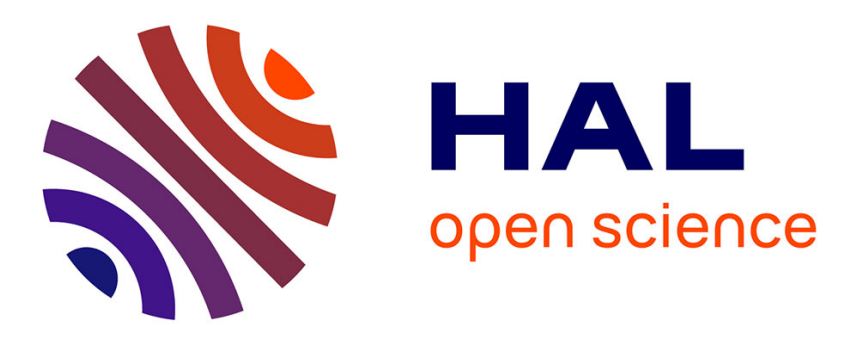

\title{
Modélisation de l'écrouissage et de la restauration en viscoplasticité cyclique
}

\author{
D. Nouailhas
}

\section{To cite this version:}

D. Nouailhas. Modélisation de l'écrouissage et de la restauration en viscoplasticité cyclique. Revue de Physique Appliquée, 1988, 23 (4), pp.339-349. 10.1051/rphysap:01988002304033900 . jpa-00245779

\section{HAL Id: jpa-00245779 https://hal.science/jpa-00245779}

Submitted on 1 Jan 1988

HAL is a multi-disciplinary open access archive for the deposit and dissemination of scientific research documents, whether they are published or not. The documents may come from teaching and research institutions in France or abroad, or from public or private research centers.
L'archive ouverte pluridisciplinaire HAL, est destinée au dépôt et à la diffusion de documents scientifiques de niveau recherche, publiés ou non, émanant des établissements d'enseignement et de recherche français ou étrangers, des laboratoires publics ou privés. 


\title{
Modélisation de l'écrouissage et de la restauration en viscoplasticité cyclique
}

\author{
D. Nouailhas
}

Office National d'Etudes et de Recherches Aérospatiales, ONERA, 92322 Châtillon Cedex, France

(Reçu le 15 juin 1987, accepté le 14 janvier 1988)

Résumé. - Ce document fait la synthèse des développements réalisés sur les lols de comportement viscoplastiques basées sur le concept de variables internes. Les effets supplémentaires d'écrouissage : mémoire de la déformation plastique, restauration, vieillissement, sont discutés sur la base de résultats expérimentaux. Les différentes variables et les lois d'évolutions associées, justifiées par les expériences existantes mais également par des considérations microstructurales, sont introduites séparément. On donne enfin, une application de ces modèles, au cas d'un acier inoxydable à haute température.

Abstract. - This paper gives a review of the developments made on viscoplastic constitutive equations based on the concept of internal variables. Additionnal effects, like plastic strain memory, aging and recovery are discussed on the basis of experimental results. Hardening variables and the associated evolution rules, justified by existing experiments but also by some microstructural considerations, are introduced independently. In the last part, an application of these models to the case of an austenitic stainless steel at elevated temperature 1s presented.

\section{Introduction.}

Les méthodes modernes de prévision de durée de vie des structures, soumises à des charges et des températures cycliques, nécessitent l'emploi de lois de comportement traduisant aussi correctement que possible le comportement réel des matériaux. Dans des situations de températures élevées, il est nécessaire de prendre en compte les effets d'écrouissage monotone et cyclique mais aussi l'influence de la vitesse et les phénomènes de restauration de la microstructure.

Les lois de comportement présentées ici, sont développées dans le cadre de la thermodynamique avec variables internes [1]. Dans ce contexte, l'état actuel du matériau dépend uniquement des valeurs actuelles d'un ensemble de variables observables et de variables internes. Ces lois sont basées sur la superposition d'un certain nombre de modèles d'écrouissage que 1 'on introduit ici dans le cadre de la viscoplasticité Cependant, le passage au cas de la plasticité indépendante du temps, qui constitue un cas limite de la viscoplasticité, se fait aisément, les mêmes variables d'écrouissage pouvant être utilisées [2] .

On présente dans ce papier une synthèse des développements réalisés sur ces lois. Après avoir rappelé la formulation classique de la viscoplasticité, basée sur la superposition d'une variable d'écrouissage cinématique non linéaire et d'une variable d'écrouissage isotrope, on montre comment des effets complexes d'écrouissage (mémoire, restauration...) peuvent être pris en compte dans ces modèles. On présente enfin, dans une dernière partie, une application au cas de 1'acier inoxydable à haute temperature, qui montre bien que les effets d'interaction plasticité-fluage observés sur ce type de matériau, peuvent être correctement décrits par une approche unifiée (une seule déformation inélastique).

\section{Viscoplasticité classique}

La loi de viscoplasticité dérive d'un potentiel viscoplastique, qui peut être particularisé par la relation [2]

$$
\Omega=\frac{K}{n+1}<\frac{J(\underset{\sigma}{\sigma}-\underset{X}{X})-R-k}{K}>^{n+1}
$$

avec les notations $\langle\mathrm{u}\rangle=\mathrm{uH}(\mathrm{u})$. H est la fonction d'Heaviside : $H(u)=0$ si $u<0 ; H(u)=1$ si $u>0$.

La vitesse d'écoulement viscoplastique s'obtient par 1'hypothèse de normalité, et dans le cas d'un matériau obéissant au critère de Von Mises on obtient la relation

$$
\underline{\varepsilon}_{p}=\frac{3}{2}<\frac{\mathrm{J}(\underline{\sigma}-\underline{\mathbf{X}})-\mathrm{R}-\mathrm{k}}{\mathrm{K}}>\frac{\mathrm{n}}{\mathrm{\sigma} \underline{\sigma}^{\prime}-\underline{X}^{\prime}}
$$

$\operatorname{avec} J(\underline{\sigma}-\underline{X})=\left[3 / 2\left(\sigma^{\prime}-\underline{X}^{\prime}\right):\left(\sigma^{\prime}-\underline{X}^{\prime}\right)\right]^{1 / 2}$

où $\sigma^{\prime}$ et $X^{\prime}$ sont les devilateurs des tenseurs $\sigma$ et X.Le sỹmbole : désigne le produit tensorifiel contracté avec sommation sur deux indices.

Ce modèle fait intervenir la notion de surface limite d'élasticité (a l'intérleur de laquelle le comportement reste élastique) et dont l'évolution est gouvernée par les variables internes d'écroulssage cinématique $\underset{X}{X}$ (position du centre) 
et isotrope $R$ (taille du rayon). Les surfaces d'égale dissipation (donc d'égale vitesse) se déduisent de cette surface seuil par homothétie, et l'on volt que le module de la vitesse est d'autant plus grand que l'état de contrainte se trouve élolgné de la surface limite d'élasticité (Fig. 1).

En traction-compression, l'equation (2) peut se mettre sous la forme :

$$
\sigma=X \pm R \pm k \pm K \dot{\varepsilon}_{p}^{1 / n}=\sigma_{i}+\sigma_{V}
$$

qui donne la décomposition de la contrainte appliquée, en une contrainte interne $\sigma_{i}=X \pm R \pm k$ et une contrainte visqueuse $\sigma_{v}=K \dot{\varepsilon}_{\mathrm{p}}^{1 / n i}$. Ceci est illustré sur la figure 2 .

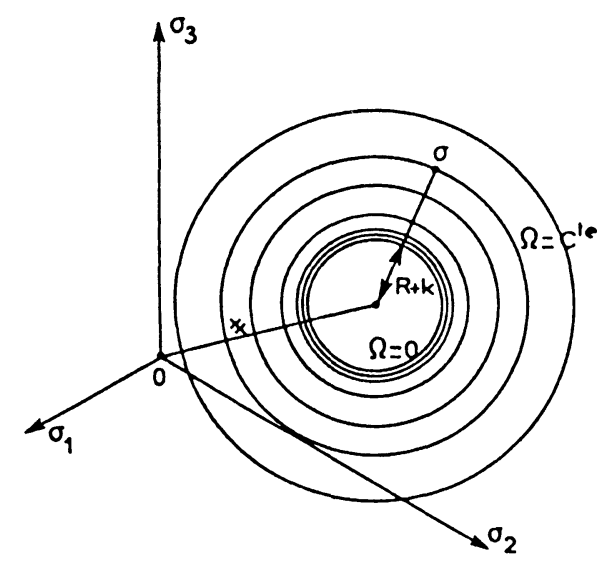

F1g. 1 - Domalne élastique et équipotentlelles en viscoplasticité.

[Elastic domain and equipotential surfaces in viscoplasticity.]

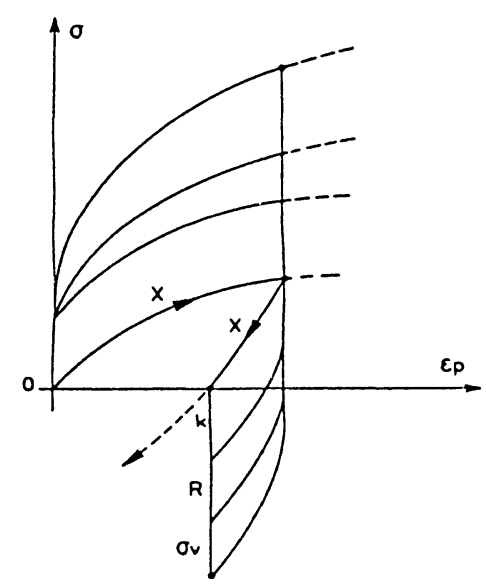

Fig. 2 - Décomposition de la contrainte appliquée. [Partition of the applied stress.]

- Le tenseur $\mathbf{X}$ correspond à la partie cinématique de la contrainte interne, mise en évidence expérimentalement [3]. Il représente la contrainte induite par les interactions à longue distance : inhomogénéités de déformations plastiques d'un grain à un autre et effets d'interaction dislocations-précipitês.

- La contrainte $k$, de nature scalaire, représente la limite initiale d'élasticité.

- La variable isotrope $R$, est introduite pour décrire le durcissement ou l'adoucissement cyclique. Elle est directement reliée à 1 'augmentation de la densité de dislocation.

- La contrainte $\sigma_{y}$, représente la partie purement visqueuse du modèle, nécessaire pour traduire l'influence de la vitesse. $K$ et $n$ sont des constantes dépendant du matériau et de la température.

2.1 ECROUISSAGE CINEMATIQUE NON LINEAIRE - Cette variable, dont la loi d'évolution constitue la loi de base du modèle cyclique, correspond à une translation des surfaces équipotentielles dans 1 'espace des contraintes. Elle est indispensable pour décrire correctement le comportement cyclique (effet Bauschinger). Par rapport à la loi linéaire développée par Prager [4], l'introduction d'un terme de rappel [5][6][7] permet d'améliorer considérablement la forme des cycles.

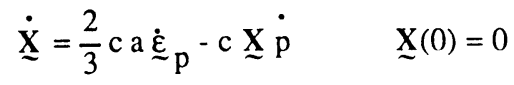

p représente la déformation plastique cumulée

$$
\dot{p}=\left[2 / 3 \dot{\dot{\varepsilon}}_{\mathrm{p}}: \dot{\dot{\varepsilon}}_{\mathrm{p}}\right]^{1 / 2}
$$

c et a sont des coefficients caractéristiques du matériau.

Dans le cas de la traction-compression, on obtient la relation

$$
\dot{X}=c a \dot{\varepsilon}_{p}-c X\left|\dot{\varepsilon}_{p}\right|
$$

qui montre clairement que la non linéarité introduite dans la loi par le terme de rappel est différente selon que l'écoulement se produit en traction ou en compression. Ceci permet de décrire correctement la concavité des boucles contrainte-deformation. Cette loi se stabilise très rapidement lors $\mathrm{d}$ 'un chargement symétrique. E1le prend alors, à chaque alternance, la valeur

$$
X= \pm a \text { th }\left(c \frac{\Delta \varepsilon}{2}\right)
$$

Le modèle cinématique non linéaire, ne conduit à 1a stabilisation des cycles, que dans les cas où le chargement est symétrique. Il permet donc qualitativement de reproduire les effets de déformation progressive (chargement avec contrainte moyenne non nulle) et de relaxation de contrainte moyenne (déformation moyenne non nulle) [8].

Dans la pratique, afin d'étendre le domaine de validité du modèle, plusieurs variables cinématiques sont introduites (géneralement deux ou trois) [9]. Ces variables sont superposées de la façon suivante :

$$
\underset{\mathbf{X}}{=} \sum_{1} \underline{\mathbf{X}}^{\mathrm{i}}
$$

Chaque variable évolue indépendament avec le même type de loi (équation 4). Une d'entre elles peut être 1inéaire : $\dot{\sim}_{k}=2 / 3 c_{k} a_{k} \dot{\dot{\varepsilon}}_{p}$

Cette extension du modèle permet également d'améliorer la description des effets de rochet. Dans le cas où une des variables est linéaire, les 
propriétés du modèle sont modifiées, et $1^{\prime}$ 'on obtient un effet de rochet transitoire suivi d'une accommodation (le modèle linéaire seul donne une accommodation immédiate) [10].

2.2 ECROUISSAGE ISOTROPE - Cette variable est introduite pour décrire le durcissement ou l'adoucissement cyclique. Son évolution est gouvernée par la déformation plastique cumulée et l'état stabilisé est atteint lorsque $R$ atteint sa valeur asymptotique $\mathrm{Q}$.

$$
\dot{\mathrm{R}}=\mathrm{b}(\mathrm{Q}-\mathrm{R}) \dot{\mathrm{p}} \quad \mathrm{R}(0)=0
$$

Les coefficients $b$ et $Q$ dépendent du matériau et de la température.

Cette loi permet de rendre compte de façon satisfaisante des essais cycliques sans temps de maintien, quelle que soit l'amplitude de déformation imposee [11], comme le montre la figure 3 qui représente l'évolution des maxima de contrainte en fonction de la déformation plastique cumulée. Lors d'un essai à déformation imposée, durant le processus de durcissement, 1'accroissement de contrainte au cours d'un cycle est approximativement Égal à 1'accroissement de la variable R. En effet, la variable cinématique (qui dépend de la déformation plastique) et la contrainte visqueuse (qui dépend de la vitesse de deformation plastique) ont une valeur à peu près constante au maximum (minimum) de chaque cycle. On peut donc assimiler, en première approximation, les variations d'amplitude de contrainte au cours d'un cycle aux variations d'écrouissage isotrope. L'intégration de la loi (7) donne :

$$
\mathrm{R}=\mathrm{Q}\left(1-\mathrm{e}^{-\mathrm{b} p}\right)
$$

Compte tenu des approximations précédentes, on peut écríre la relation :

$$
\frac{\sigma_{M}-\sigma_{M_{O}}}{\sigma_{M_{S}}-\sigma_{M_{O}}} \approx \frac{R}{Q}=1-e^{-b p}
$$

avec $\sigma_{\mathrm{M}}, \sigma_{\mathrm{M}}$ et $\sigma_{\mathrm{M} S}$ respectivement les maxima de contrainte, ${ }^{O}$ ses valeurs au premier cycle et au cycle stabilisé.

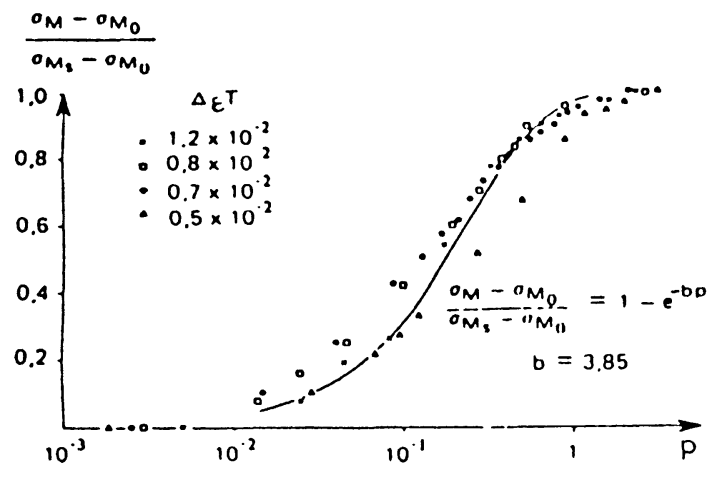

Fig. 3 - Evolution de $1^{\star}$ écrouissage isotrope en fonction de la déformation plastique cumulée (acier inoxydable 316 à $600^{\circ} \mathrm{C}$ ).

[Isotropic hardening vs. accumulated plastic strain for $316 \mathrm{~S} . \mathrm{S}$ at $600^{\circ} \mathrm{C}$.]
Cette interprétation des essais, constitue un moyen d'identifier le coefficient b.

Etant donné le nombre de cycles généralement nécessaire pour atteindre les conditions stabilisées (de 1 'ordre de 20 à 100 cycles selon 1 'amplitude de déformation), l'évolution de la variable isotrope est beaucoup plus lente que celle de la variable cinématique (le coefficient b de 1 'équation (7) est beaucoup plus petit que le coefficient c de l'équation (4)). Mais dans l'état stabilisé (écrouissage isotrope saturé), le seul effet d'écrouissage qui subsiste est cinématique.

La comparaison entre la courbe monotone et la courbe cyclique s'obtient immédiatement en utilisant l'équation (3).

Traction monotone :

$$
\sigma=\mathrm{a}\left(1-\mathrm{e}^{-c \varepsilon_{\mathrm{p}}}\right)+\mathrm{Q}\left(1-\mathrm{e}^{-\mathrm{b} \varepsilon_{\mathrm{p}}}\right)+\mathrm{k}+\mathrm{K} \dot{\varepsilon}_{\mathrm{p}}^{1 / \mathrm{n}}
$$

courbe cyclique :

$$
\frac{\Delta \sigma}{2}=a \text { th }\left(\mathrm{c} \frac{\Delta \varepsilon_{\mathrm{p}}}{2}\right)+\mathrm{Q}+\mathrm{k}+\mathrm{K} \varepsilon_{\mathrm{p}}^{1 / \mathrm{n}}
$$

Pour des essais réalisés à 1 a même vitesse de déformation, la différence entre $\sigma$ et $\Delta \sigma / 2$ est à peu près égale à $Q$. En effet, le premier terme des deux équations est très peu différent, et 1'écrouissage isotrope développé pendant la traction monotone est très faible (pour de petites déformations). La courbe d'écrouissage cyclique est donc approximativement parallèle à la courbe monotone.

L'écrouissage isotrope, qui est introdult ici de façon totalement indépendante, peut également intervenir sur la variable d'écrouissage cinématique et sur la contrainte visqueuse.

On observe pour certains matériaux, une variation progressive du module tangent d'un cycle à 1'autre. Ceci peut être décrit par l'introduction d'une fonction $\phi(p)$ dans la loi d'évolution de la variable cinématique (équation 4) [6].

$$
\underset{\mathrm{X}}{\dot{\mathrm{X}}}=\frac{2}{3} \mathrm{c} \mathrm{a}{\underset{\mathrm{\varepsilon}}{\mathrm{p}}}_{\mathrm{p}}-\mathrm{c} \phi(\mathrm{p}) \underset{\mathrm{X}}{\dot{\mathrm{p}}}
$$

avec

$$
\phi(p)=\phi_{S}+\left(1-\phi_{S}\right) e^{-b p}
$$

L'introduction de 1 'écrouissage isotrope dans la contrainte visqueuse, permet également d'améliorer la description des essais de relaxation cyclique, où l'on observe que la quantité de contrainte relaxée à chaque cycle augmente en même temps que l'amplitude de contrainte [11]. Ce couplage peut être introduit dans le modèle par une relation de la forme :

$$
\mathrm{K}=\mathrm{K}_{\mathrm{O}}+\alpha_{\mathrm{K}} \mathrm{R}
$$

où $\alpha_{K}$ est un coefficient supplémentaire.

3. Modélisation d'effets complexes.

Les modèles décrits ci-dessus, utilisant une superposition d'écroulssage isotrope et d'écrouissage cinématique non linéaire ont été appliqués avec succès à différents matériaux : IN 100, INCO 718, TA6V. Cependant, pour certains matériaux et selon le chargement appliqué, on observe expérimentalement des effets complexes d'écrouissage. Etant dus à des phénomènes physiques supplémentaires, ils mettent en défaut le modèle de base : effets de mémoire de la déformation plastique, effets de restauration ou de vieillissement. 
3.1 INFLUENCE DE LA DEFORMATION -

- Mémoire de 1'écrouissage

Cet effet de mémoire est $\mathrm{mls}$ en évidence par differents types d'essais cycliques [12] : essais à plusieurs niveaux (croissants ou décroissants), essais incrémentaux, qui montrent que la courbe d'écrouissage cyclique dépend de la procédure expérimentale. Dans le cas de 1 'acier 316 à température ambiante, on observe le comportement suivant :

- Après saturation de 1'écroulssage cyclique pour une amplitude de deformation donnée, plusieurs cycles sont nécessaires pour atteindre un nouvel état stabilisé lorsqu'on augmente 1'amplitude de déformation. Mais on retrouve une courbe cyclique identique à celle obtenue de façon classique (une seule amplitude par éprouvette).

- Dans un essai à niveaux décroissants, après cyclage au niveau le plus élevé, l'amplitude de contrainte à niveau plus faible est très largement supérieure à celle obtenue lorsqu'il n'y a pas de précyclage. I1 y a donc mémorisation de la déformation antérieure, mais cette mémoire est partie1lement évanescente ce qui se manifeste par un lent adoucissement lorsqu'on poursuit le cyclage à faible niveau (Fig. 4). Mais 11 faut remarquer que deux échelles distinctes interviennent dans ce processus de mémorisation, car si une vingtaine de cycles suffisent pour mémoriser le premier chargement, plusieurs centaines sont ensuite nécessaires pour l'oublier partiellement.

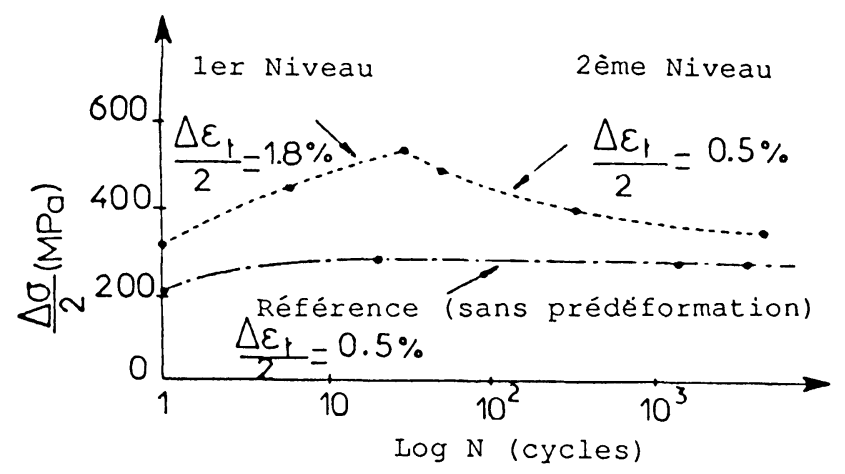

Fig. 4 - Evolution de l'amplitude de contrainte dans un essal à niveaux décrolssants $\left(316\right.$ a $20^{\circ} \mathrm{C}$ ).

[Evolution of the stress range during a decreasing two-leve1 test on 316 SS at room temperature.]

- L'effet de mémorisation est également observé dans les essais incrémentaux, où l'amplitude de déformation imposée est successivement croissante puis décroissante.

- Une des conséquences de l'effet de mémoire est 1a très grande différence de forme entre la courbe monotone, la courbe cyclique (Fig. 6) et les boucles d'hystérésis. En particulier, la règle de Masing n'est pas vérifié.

La loi d'écrouissage isotrope, introdulte précédemment ne permet pas de décrire ces effets puisqu'elle conduit, sous conditions stabilisées, à une valeur unique de durcissement cyclique, quelle que soit $1^{\prime}$ histoire de la déformation.

Les effets de mémoire de la déformation sont introduits dans le modèle par l'intermédiaire d'une nouvelle variable d'écrouissage q [11]. D'un point de vue physique, des analyses de microstructures montrent que cette variable peut être associée à la présence de cellules de dislocation dont la taille et l'épaisseur des parois dépendent de 1'amplitude de déformation. La création de macles à niveau élevé, quil persistent quel que soit le cyclage ultérieur correspond à la partie non évenescente de 1 'effet de mémoire [13]

Dans ce qui suit, on présente le modèle à mémoire complète [12]. Mais la prise en compte de 1 'évanescence, qui ne constitue pas le phénomène prépondérant, peut se faire aisément en modifiant la loi d'évolution de la variable q [14].

La formulation générale consiste à introduire, dans l'espace des deformations plastiques, une surface de non-écrouissage

$$
\begin{aligned}
& F=I\left(\varepsilon_{p}-\xi\right)-q \leq 0 \\
& I\left(\underline{\varepsilon}_{p}-\underline{\xi}\right)=\left[\frac{2}{3}\left(\underline{\varepsilon}_{p}-\underline{\xi}\right):\left(\underline{\varepsilon}_{p}-\underline{\xi}\right)\right]^{1 / 2}
\end{aligned}
$$

L'évolution des variables $\xi$ et $q$, qui représentent respectivement le centre de $1 \mathrm{la}$ surface et son rayon, n'est possible que si $F=0$. L'évolution du rayon est donnée par la relation

$$
\dot{\mathrm{q}}=\eta \mathrm{H}(\mathrm{F})\left(\underline{\mathbf{n}}: \underline{\mathbf{n}}^{*}\right) \dot{\mathrm{p}}
$$

où $\underset{\mathbf{n}}{\sim}$ et $\underline{\mathbf{n}}^{*}$ sont les normales unitaires à $1 \mathrm{a}$ surface d'élasticité et la surface mémoire (F) aux points $\underline{\sigma}$ et $\underline{\mathcal{E}}_{\mathrm{p}}$. La condition de consistence $(F=\dot{F}=0)$ impose la loi d'évolution du centre $\xi$

$$
\dot{\xi}=\sqrt{\frac{3}{2}}(1-\eta) H(F)<\underline{n}: \underline{n}^{*}>\underline{n}^{*} \dot{p}
$$

Le coefficient $\eta$ donne la répartition entre la partie cinématique de la mémoire et la partie isotrope. $\eta=1 / 2$ correspond au modèle initialement développé par Chaboche et a1. [12], mais Ohno [15] et Dafalias [16] ont montré qu'une valeur inferieure pour $\eta$, introduit un effet de progressivité dans l'établissement de la mémoire, ce qui conduit à de meilleurs résultats pour des chargements plus complexes.

L'effet de mémoire est introduit dans $1 \mathrm{a}$ lo1 d'écrouissage isotrope, en faisant dépendre la valeur asymptotique $Q$ de la variable $q$. On a donc toujours la relation :

$$
\dot{\mathrm{R}}=\mathrm{b}(\mathrm{Q}-\mathrm{R}) \dot{\mathrm{p}} \quad \mathrm{Q}(0)=\mathrm{Q}_{\mathrm{O}}
$$

avec

$$
\dot{\mathrm{Q}}=2 \mu\left(\mathrm{Q}_{\max }-\mathrm{Q}\right) \dot{\mathrm{q}}
$$

Dans le cas de la traction-compression, $q$ est égal à $\Delta \varepsilon_{\mathrm{p}} / 2,1$ 'amplitude de déformation plastique du cycle où la mémoire est stabilisée (si $\eta$ $=1 / 2, q=\Delta \varepsilon_{\mathrm{p} \text { max }} / 2$ car la mémoire est. stabilisée en un cycle dans ce cas).

On voit donc que la valeur asymptotique de $1 \mathrm{a}$ variable isotrope dépend de l'amplitude de déformation plastique :

$$
\mathrm{Q}=\mathrm{Q}_{\max }+\left(\mathrm{Q}_{\mathrm{O}}-\mathrm{Q}_{\max }\right) \mathrm{e}^{-\mu \Delta \varepsilon_{\mathrm{p}}}
$$

Ce modèle, appliqué à 1 'acier 316 , permet de décrire correctement 1 'ensemble des effets expérimentaux : la courbe monotone, les diverses courbes cycliques, les cycles successifs pour plusieurs amplitudes de déformation, comme le montrent les figures 5 et 6 .

- Chargements non proportionnels.

Les nombreux essais réalisés ces dernières 
années sous chargements de traction-torsion, montrent qu'un chargement hors phase prodult un durcissement beaucoup plus important que celui obtenu sous chargement proportionnel à même amplitude de déformation équivalente. Cet effet, observé sur de nombreux matériaux qui se durcissent cycliquement [17], [18], [19], se manifeste également, de façon moins spectaculaire cependant, sur un matériau à adoucissement cyclique tel que 1'INCO 718 [20].

Les résultats obtenus sur 1 'acier 316 à température ambiante, peuvent se résumer de $1 \mathrm{a}$ façon suivante [21]:

- Le durcissement supplémentaire produit par le chargement hors phase, est d'autant plus important que le déphasage entre la déformation axiale et la déformation de torsion est grand.
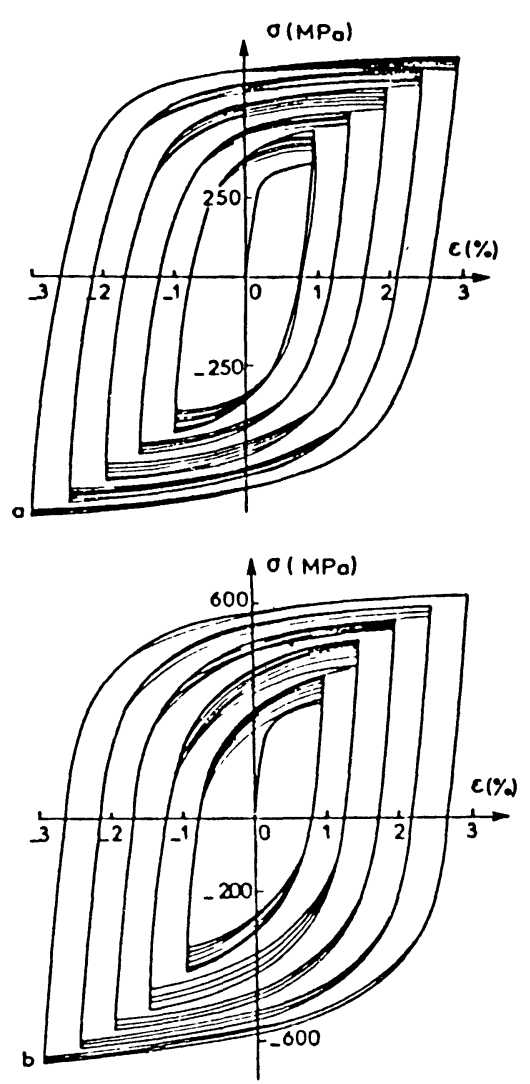

F1g. 5 - Essal à niveaux crolssants sur du 316 L [12] : a) expérience; b) simulation.

[Cyclic test at increasing levels on 316 sS : a) experiment ; b) simulation.]

- A chaque changement de direction de cyclage (traction puis torsion ou l'inverse) un durcissement supplémentaire est observé dès le premier cycle et est suivi d'un lent adoucissement.

Ces effets, interprétés physiquement à travers le concept d'écrouissage latent, peuvent être introduits dans les modeles utilisant une surface seuil, de deux façons :

- En introduisant une évolution anisotrope de la surface seuil (anisotropie induite par le chargement ) [22].

- En modifiant la loi d'écroulssage isotrope, par 1'intermédiaire d'une nouvelle variable [21] [23].

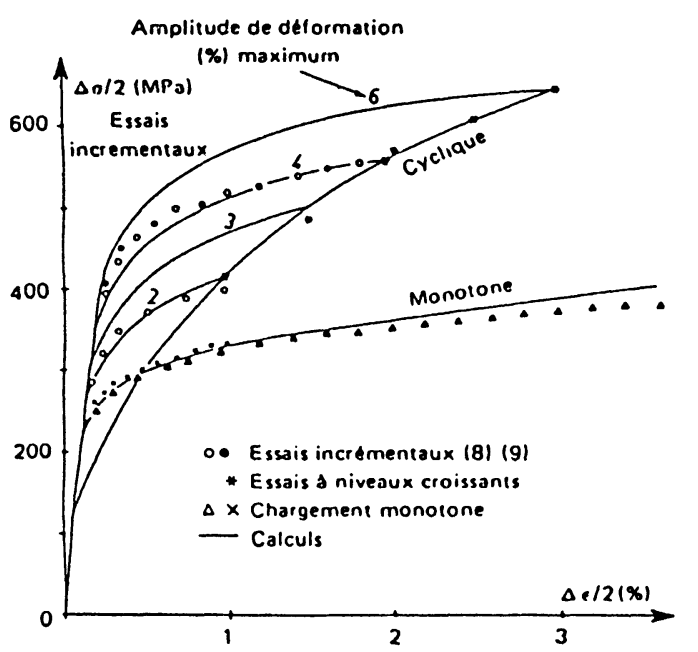

Fig. 6 - Prédiction des essals Incrémentaux sur du 316 L [12].

[Prediction of an incremental test on $316 \mathrm{SS} \cdot]$

3.2 INFLUENCE DU TEMPS - A haute température, lorsque les chargements font intervenir des séquences de temps de maintien, on observe sur les aciers inoxydables de type 304 ou 316 des effets du temps qui peuvent être soit des effets de restauration, soit des effets de vieillissement.

- Effets de restauration

Ces effets de restauration, provoqués par des phénomènes d'agitation thermique ou des effets de recristallisation, se traduisent macroscopiquement par une diminution avec le temps de l'écrouissage produit initialement par la déformation.

De nombreux essais, monotones et cycliques, réalisés sur des aciers inoxydables révèlent la présence d'effets de restauration.

- Un maintien à force nulle, après un écrouissage monotone ou cyclique [25], conduit à une diminution de la limite d'écoulement lors d'un nouveau chargement de traction (Fig. 7).

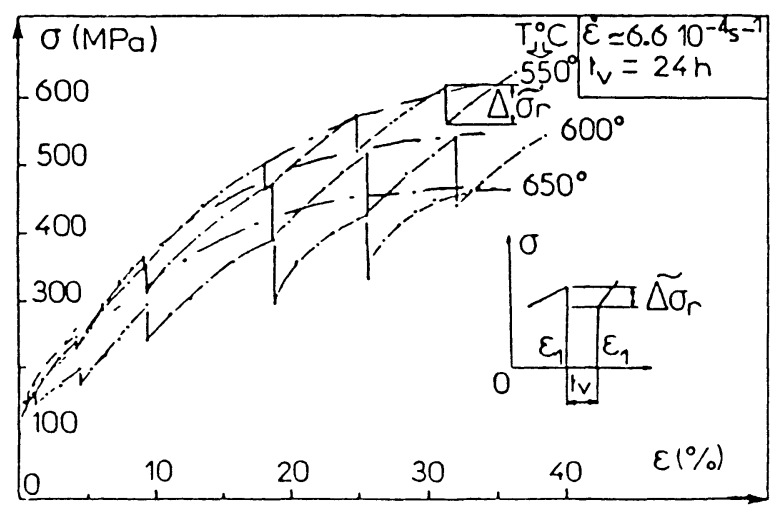

Fig. 7 - Mise en évidence de la restauration au cours d'un ma1ntien de 24 h. sur du 316 [24].

[Time recovery effect on the monotonic tensile curve.] 
- Des essais cycliques avec temps de maintien en traction ne conduisent pas à une courbe cyclique unique [11] [25]. Pour une amplitude de déformation donnée, la contrainte maximale au cycle stabilisé diminue lorsque le temps de maintien augmente. Cette diminution de l'amplitude de contrainte est due à une diminution de la taille du domaine d'élasticité, donc de l'écrouissage isotrope, comme le montre la figure 8.

- La présence d'un temps de maintien au maximum du cycle, modifie la concavité de 1 a branche du cycle correspondant à la décharge, comme le montre 1a figure 9. On observe que l'état d'écrouissage est plus faible à la fin de la relaxation qu'au début. Cette dissymétrie de la boucle d'hystérésis ne

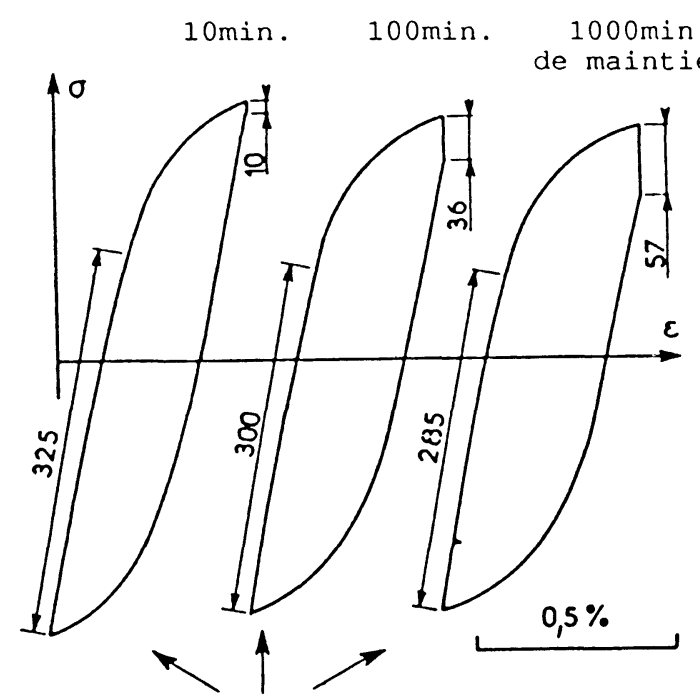

cycles stabilisés

Fig. 8 - Influence du temps de maintien sur l'amplitude de contrainte $\left(316\right.$ a $\left.600^{\circ} \mathrm{C}[11]\right)$.

[Effect of hold time in tension on the stress range in the stabilized state $\left(316 \mathrm{SS}, 600^{\circ} \mathrm{C}\right) \cdot$ ]

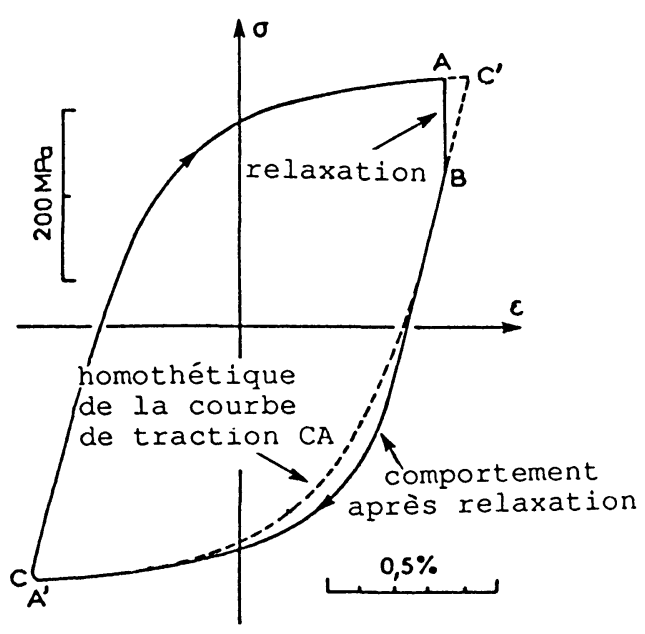

Fig. 9 - Influence d'un maintien de $24 \mathrm{~h}$ sur l'écoulement plastique en compression ( 316 à $600^{\circ} \mathrm{C}$ ).

[Effect of hold time in tension on the resulting plastic flow in compression.] peut être décrite que par un effet de restauration de la variable cinématique, l'évolution de 1 a variable isotrope étant beaucoup trop faible au cours d'un cycle.

Un terme de restauration est introduit dans chacune des lois d'évolutions des variables d'écrouissage cinématique et isotrope de la façon suivante [2]

$$
\underset{\sim}{\dot{X}}=\frac{2}{3} \mathrm{ca}{\underset{\mathrm{\varepsilon}}{\mathrm{p}}}_{\mathrm{d}}-\mathrm{c} \underset{\sim}{\mathrm{X}} \dot{\mathrm{p}}-\beta\left|\mathrm{X}_{\mathrm{II}}\right|^{\mathrm{r}-1} \underset{\sim}{\mathrm{X}}
$$

avec $\mathrm{X}_{\mathrm{II}}=\left[\frac{2}{3} \underset{\sim}{\mathrm{X}}: \underset{\mathrm{X}}{\mathrm{X}}\right]^{1 / 2}$

$$
\dot{R}=b(Q-R) \dot{p}+\gamma\left|Q_{r}-R\right|^{m-1}\left(Q_{r}-R\right)
$$

Les coefficients $\beta, r, \gamma$ et $m$ dépendent fortement de la température.

Dans 1'équation (21), 1'effet de restauration n'est que partiel. La valeur asymptotique $Q_{r}$, dépend comme $Q$, de la mémoire de la déformation plastique, afin de prendre en compte le fait que la restauration de la microstructure est différente avant et après un écrouissage cyclique [26].

$$
\mathrm{Q}_{\mathrm{r}}=\mathrm{Q}-\mathrm{Q}_{\mathrm{r}}^{*}\left[1-\left(\frac{\mathrm{Q}_{\max }-\mathrm{Q}}{\mathrm{Q}_{\max }}\right)^{2}\right]
$$

Dans le cas d'un matériau sans mémoire, $Q_{R}$ est une constante.

Dans l'état initial $R=0<Q_{r}<Q$. Sous chargement cyclique, le modèle donne toujours un effet de restauration, la valeur de $R$ devenant toujours supérieure à $Q_{r}$ après quelques cycles. Pour une amplitude de déformation donnée, la valeur stabilisée de $R\left(Q_{r} \leq R_{S} \leq Q\right)$ est d'autant plus faible que le maintien est long. Sous chargement monotone, selon les valeurs respectives de $R$ et de $Q_{r}$ le modèle donne soit un effet de viefllissement (augmentation de $\mathrm{R}$ en fonction du temps), soit un effet de restauration. Ce point sera repris plus loin.

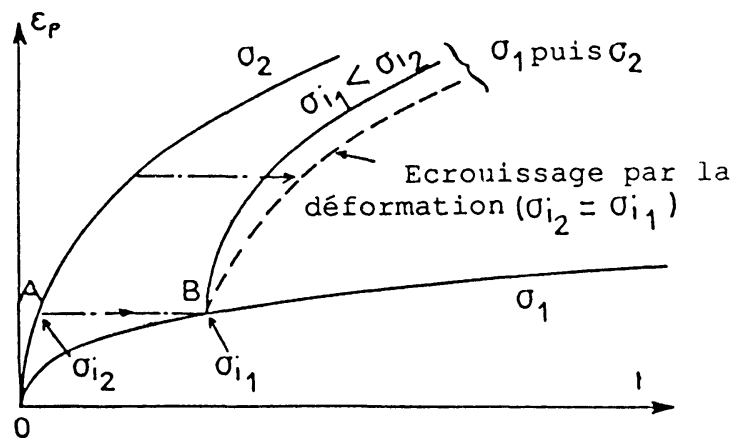

F1g. 10 - Effet de la restauration sur le fluage au second niveau.

[Effect of time recovery on the second creep leve1.]

Les termes de restauration par le temps permettent également de traduire les effets d'accélération et d'hésitation au fluage dans des essais de fluage à deux niveaux [27], [28]. Ceci est illustré sur la figure 10 dans le cas d'un essai à niveaux croissants. L'accélération au second niveau, par rapport à l'hypothèse d'écrouissage 
par la déformation, est due au fait que la contrainte interne $\sigma_{i 1}$ au point $B$ est plus faible que la contrainte $\sigma_{12}$ au point $A$, à cause de 1 'effet de restauration.

- Effets de vieillissement

Un vieillissement initial (sans déformation) provoque generalement une augmentation de la résistance à la déformation, ce qui condult à des courbes de traction ou de fluage differrentes [29]. Le même effet peut être observé après des essais de fluage de longue durée (Fig. 11) [30].

Cet effet, lié à des phénomènes de précipitation, peut être décrit par une combinaison

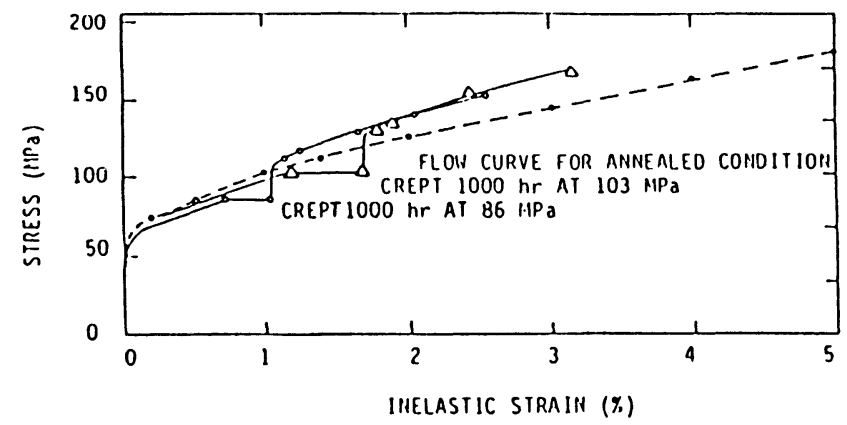

F1g. 11 - Influence du fluage sur la courbe de traction $\left(304\right.$ a $\left.593^{\circ} \mathrm{C}[30]\right)$.

[Interaction between creep and plasticity on 304 SS at $\left.593^{\circ} \mathrm{C}.\right]$

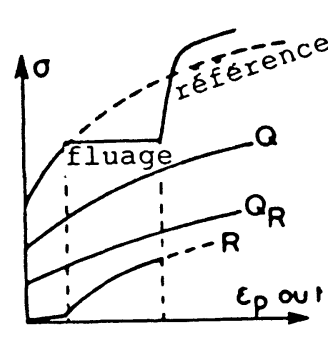

$\mathrm{R}<\mathrm{Qr}<\mathrm{Q}$

a-vieillissement sous charge

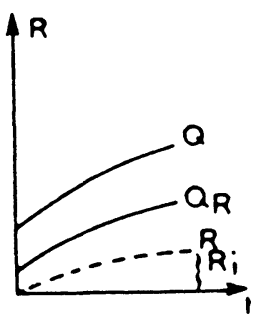

$\mathrm{R}<\mathrm{Qr}<\mathrm{Q}$

c-vieillissement initial

Fig. 12 - Evolution schématique de l'écroulssage isotrope en fonction du chargement.

[Schematical evolution of the istropic hardening variable for various loading conditions.]

d'écrouissage par la dêformation et d'écrouissage par le temps, telle qu'elle a été introduite dans 1 'équation (21).

Les possibilités du modèle sont illustrées schématiquement sur la figure 12. L'effet de vieillissement est obtenu tant que $\mathrm{R}$ reste inférieur $\mathrm{a}$
$\mathrm{Q}_{\mathrm{r}}$. Dans ce cas, la variable d'écrouissage isotrope augmente à la fois par le temps et la déformation plastique.

3.3 INFLUENCE DE LA VITESSE - I1 ne $s$ 'agit pas ici de l'effet de viscosité classique parfaitement bien décrit par une fonction puissance telle que 1 'équation (1), mais du phénomène de vieillissement dynamique, qui correspond à une instabilité de microstructure. Ce phénomène, qui se produit pour les aciers inoxydables dans un domaine de

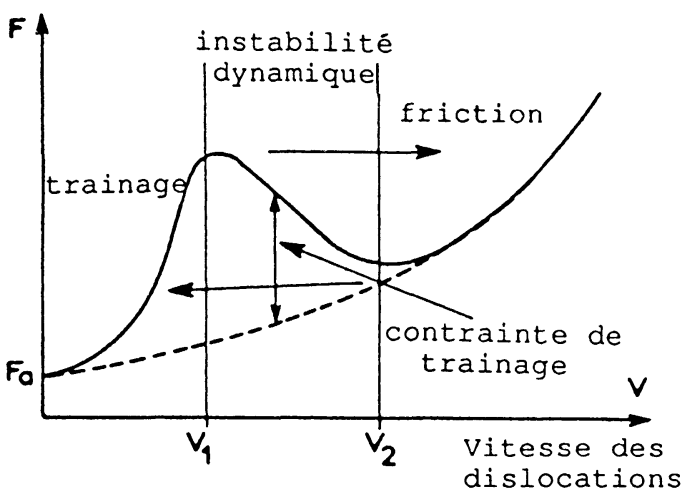

Fig. 13 - Représentation schématique des deux mécanismes intervenant dans l'effet de viefl11ssement dynamique.

[Schematical representation of the mechanisms leading to dynamical ageing effect.]

température intermédiaire (400 à $600^{\circ} \mathrm{C}$ environ), résulte d'une compétition entre deux modes de déformation : trainage et friction [31]. Ceci est illustré schématiquement sur la figure 13 : les dislocations, freinées par un mécanisme de trainée sont désancrées lorsque la contrainte atteint une valeur supérieure à la contrainte de trainage et prennent alors une vitesse très rapide. Elles sont ensuite freinées par la force de frottement. Ces deux modes de deformation se succèdent alternativement et conduisent aux instabilités caractéristiques de l'effet Portevin Le Chatelier.

On observe également, dans le domaine d'existence de ce phénomène, que les déformations importantes qui se produisent à la mise en charge, ne

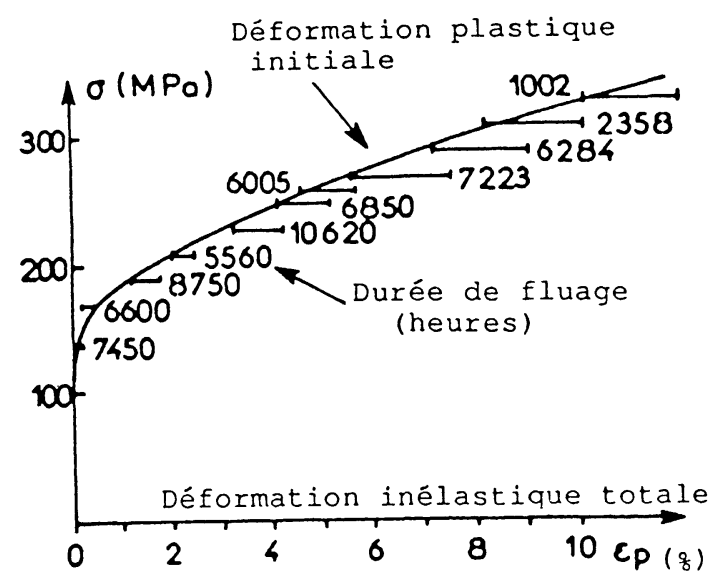

F1g. 14 - Déformation de mise en charge et de fluage sur un acler 316 à $550^{\circ} \mathrm{C}$.

[Initial plastic strains and subsequent creep strains on $316 \mathrm{SS}$ at $550^{\circ} \mathrm{C}$.] 
sont suivies que par des déformations de fluage très faibles, même en un temps très long (Fig. 14). Ceci peut s'expliquer par le passage du mode de frottement (mise en charge rapide) au mode de trainage en début de fluage (diminution de la vitesse).

Ceci est également en accord avec les courbes isochrones du code case, qui tendent vers une courbe unique lorsque le temps augmente. Pour des températures supérieures ou inférieures, cet effet de saturation n'apparaft pas, et l'on obtient un comportement viscoplastique "classique".

L'introduction d'une fonction exponentielle dans le potentiel viscoplastique permet de décrire 1 'effet de saturation de 1 'influence de la vitesse de déformation.

$$
\begin{gathered}
\Omega=\frac{\mathrm{K}}{\alpha(\mathrm{n}+1)} \exp \left(\alpha<\frac{\sigma_{\mathrm{V}}}{\mathrm{K}}>^{\mathrm{n}+1}\right) \\
\sigma_{\mathrm{V}}=\mathrm{J}(\underline{\sigma}-\underset{\mathbf{X}}{\mathbf{X}}-\mathrm{R}-\mathrm{k}
\end{gathered}
$$

Le module de la vitesse de déformation plastique vaut alors :

$$
\left.\dot{\mathrm{p}}=\left\langle\frac{\sigma_{\mathrm{v}}}{\mathrm{K}}\right\rangle^{\mathrm{n}} \exp \left(\alpha<\frac{\sigma_{\mathrm{v}}}{\mathrm{K}}\right\rangle^{\mathrm{n}+1}\right)
$$

L'évolution de la contrainte visqueuse en fonction de la vitesse de déformation plastique est représentée sur la figure 15 . On voit que cette formulation revient à introduire un exposant de viscosité variable, variant de $\mathrm{n}$ pour les faibles contraintes, à l'infini. Dans le domaine des grandes vitesses, défini par la valeur du coefficient $\alpha$, le modèle donne un comportement quasiment indépendant du temps. Les propriétés induites par cette modification sont très similaires à celles du modèle développé par Miller [32] avec des expressions analytiques différentes. Dans le modèle de Delobelle [24], le même principe est utilisé, avec une formulation différente.

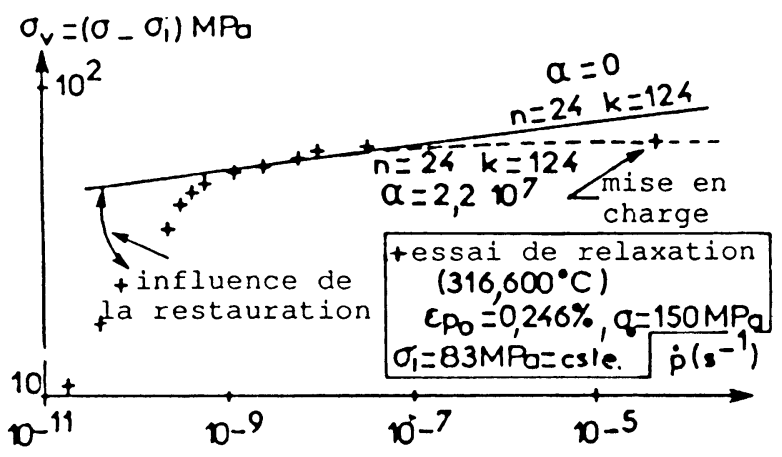

F1g. 15 - Evolution de la contrainte visqueuse en fonction de la vitesse.

[Viscous stress vs. plastic strain rate.]

\section{Modélisation unifiée.}

Dans le modèle de viscoplasticité le plus général, tous les développements présentés ici peuvent coexister. Les différentes composantes de ce modèle sont résumées dans le tableau I, où $1^{\prime}$ on précise également les variables qui peuvent être utilisées dans un schéma de plasticité.

L'utilisation, donc l'identification, de l'ensemble de la loj de comportement n'est cependant pas systématiquement nécessaire pour toutes les applications. Le choix des différentes composantes dépend du matériau, du domaine de température, du chargement et enfin de la finalité du calcul.

En ce qui concerne l'identification des différents coefficients, 1a plupart des effets mentionnés peuvent être identifiés séparément, même s'ils existent simultanément, en utilisant des expériences spécifiques.

\section{Application à 1'acier 23CND 17-12.}

On montre dans ce qui suit, une application au cas de $1^{\prime}$ acier inoxydable Z3CND $17-12$ à $600^{\circ}$. Toutes les possibilités du modèle sont utilisées, à l'exception de l'influence de la non proportionnalité, tous les chargements étudiés ici étant uniaxiaux. L'ensemble des équations (en traction-compression) ainsi que les valeurs des différents coefficients sont données dans les tableaux II et III [33]. Ces coefficients ont été identifiés à partir d'essais provenant d'EdF : essais de relaxation, essais de fluage où la déformation totale n'excedait pas $6 \%$, essais cycliques à $\pm 0,6 \%$ de déformation avec et sans temps de maiñtien.

On montre sur les figures qui suivent, une illustration de quelques possibilités du modèle, à 1 'aide de prévisions d'essais monotones et cycliques provenant de differents laboratoires.

Les figures 16 et 17 illustrent le cas des chargements de fluage (à niveaux variables) et de relaxation. Malgré les déformations importantes, produites dans ces essais $(9,5 \%$ de déformation de mise en charge dans $1^{\prime}$ essai de relaxation), très supérieures à celles du domaine d'identification des coefficients, les résultats des calculs sont en bon accord avec les expériences.

L'effet de restauration par le temps est également correctement pris en compte par le modèle. Un exemple en est donné sur la figure 18 qui reproduit l'influence d'un temps de maintien de 24 heures sur la courbe de traction, après une consolidation cyclique initiale.

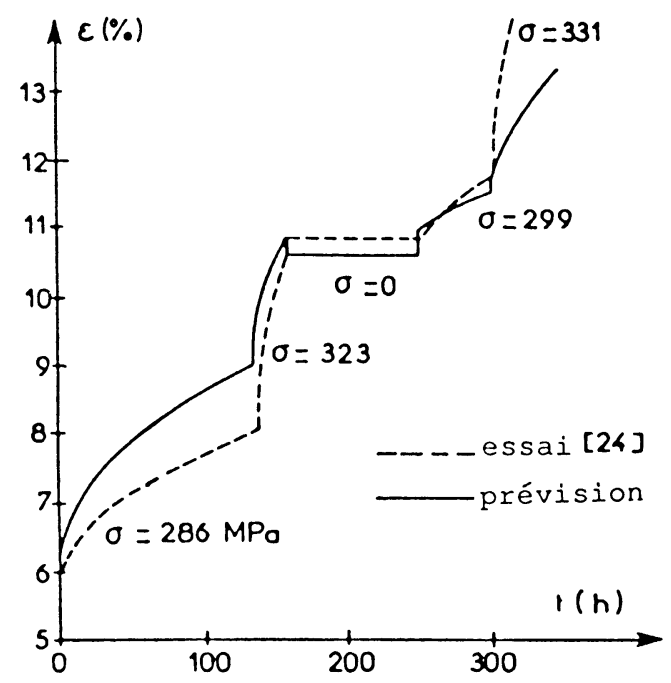

F1g. 16 - Prévision d'un essal de fluage à niveaux variables.

[Creep tests at various levels. Data and prediction.] 


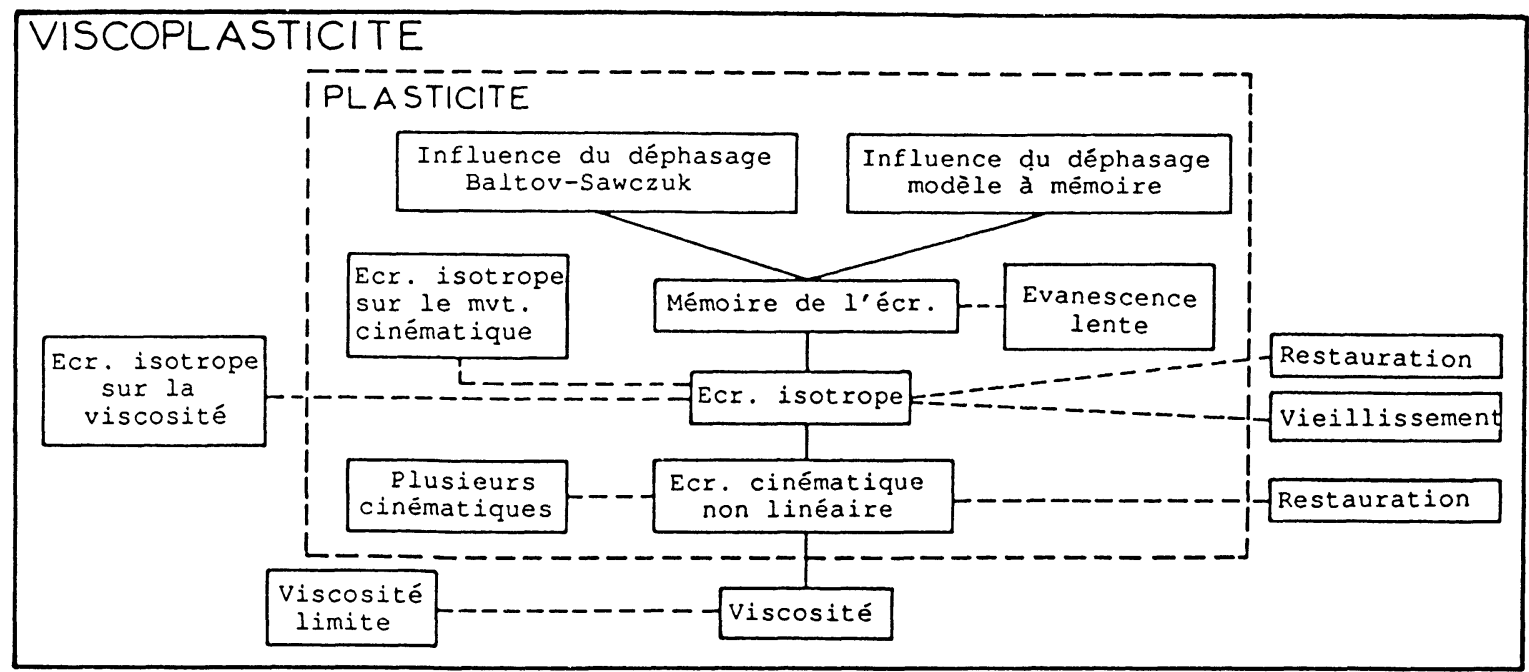

Tableau I - Synthèse des composantes du modèle.

[Synthesis of the components of the model.]

$$
\begin{aligned}
& \left.\varepsilon_{p}=\left\langle\frac{\sigma_{v}}{K}\right\rangle^{n} \exp \left(\alpha<\frac{\sigma_{v}}{k}\right\rangle^{n+1}\right) \operatorname{sgn}(\sigma-X) \\
& \sigma_{\mathrm{V}}=|\sigma-\mathrm{X}|-\mathrm{R}^{*}-\mathrm{k} \\
& \dot{X}_{1}=c_{1}\left(a_{1} \dot{\varepsilon}_{p}-\phi(p) X_{1} \dot{p}\right)-\beta_{1}\left|X_{1}\right|^{r_{1}-1} X_{1} \\
& \dot{x}_{2}=c_{2}\left(a_{2} \dot{\varepsilon}_{p}-\phi(p) x_{2} \dot{p}\right)-\beta_{2}\left|x_{2}\right|^{r_{2}-1} x_{2} \\
& \phi(p)=\phi_{S}+\left(1-\phi_{S}\right) e^{-b p} \\
& \dot{\mathrm{R}}=\mathrm{b}(\mathrm{Q}-\mathrm{R}) \dot{\mathrm{p}}+\gamma\left|\mathrm{Q}_{\mathrm{r}}-\mathrm{R}\right|^{\mathrm{m}-1}\left(\mathrm{Q}_{\mathrm{r}}-\mathrm{R}\right) \\
& \mathrm{K}(\mathrm{R})=\mathrm{K}_{\mathrm{O}}+\alpha_{\mathrm{K}} \mathrm{R} \quad ; \quad \mathrm{R}^{*}=\alpha_{\mathrm{R}} \mathrm{R} \\
& \dot{\mathrm{Q}}=2 \mu \eta \mathrm{H}(\mathrm{F})\left(\mathrm{Q}_{\max }-\mathrm{Q}\right) \dot{\mathrm{p}} \\
& I(F)=\left|\varepsilon_{p}-\xi\right|-c \leq 0 \\
& \dot{q}=\eta H(F) \dot{p} \quad, \quad \dot{\xi}=(1-\eta) H(F) \dot{\varepsilon}_{p} \\
& Q_{r}=Q-Q_{r}^{*}\left[1-\left(\frac{Q_{\max }-Q}{Q_{\max }}\right)^{2}\right]
\end{aligned}
$$

Tableau II - Ensemble des équations de la lo1 de 1 'acier 23 CND 17-12.

[Set of constitutive equations appropriate for the description of austenitic stainless steel.]

L'ensemble des caractéristiques du comportement cyclique est rassemblé sur la figure 19 . $\sigma_{\text {tmax }}$ et $\sigma$ min représentent respectivement la contrainte maximale du cycle stabilisé et la contrainte à la fin du temps de maintien en traction. La chute de 1a contrainte $\sigma_{t} \max$ lorsque le temps de maintien augmente est due à la restauration de la variabie isotrope. L'augmentation de la quantité relaxée $\left(\sigma_{t \max }-\sigma_{t} \min \right)$ est due bien sûr à 1 'influence de la viscosité, mais également à la restauration de la variable cirématique.

\begin{tabular}{|lll|}
\hline$\alpha=210^{6}$ & $\mathrm{n}=24$ & $\mathrm{k}=10$ \\
$\mathrm{~K}_{0}=116$ & $\alpha_{\mathrm{K}}=2$ & $\alpha_{\mathrm{R}}=0$ \\
$\mathrm{c}_{1}=45$ & $\mathrm{a}_{1}=80$ & $\phi_{\mathrm{S}}=0.5$ \\
$\mathrm{c}_{2}=1300$ & $\mathrm{a}_{2}=67.5$ & $\mathrm{~b}=12$ \\
$\beta_{1}=0.510^{-14}$ & $\beta_{2}=0.910^{-11}$ & $\mathrm{r}_{1}=\mathrm{r}_{2}=4$ \\
$\gamma=0.210^{-6}$ & $\mathrm{~m}=2$ & $\eta=0.6$ \\
$\mu=19$ & $\mathrm{Q}_{0}=30$ & $\mathrm{Q}_{\max }=455$ \\
$\mathrm{Q}_{\mathrm{r}}^{*}=200$ & & \\
\hline
\end{tabular}

Tableau III - Coefficients pour $1^{\prime}$ acier 23 CND $17-12$ à $600^{\circ} \mathrm{C}$.

[Material constants for Z3 CND 17-12 stainless stee 1 at $600^{\circ} \mathrm{C}$.]

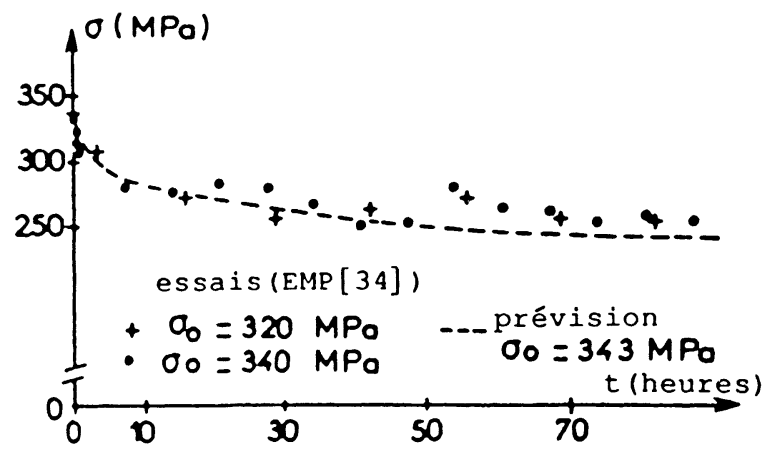

F1g. 17 - Prévision d'un essal de relaxation.

[Data and prediction of stress relaxation tests.] 


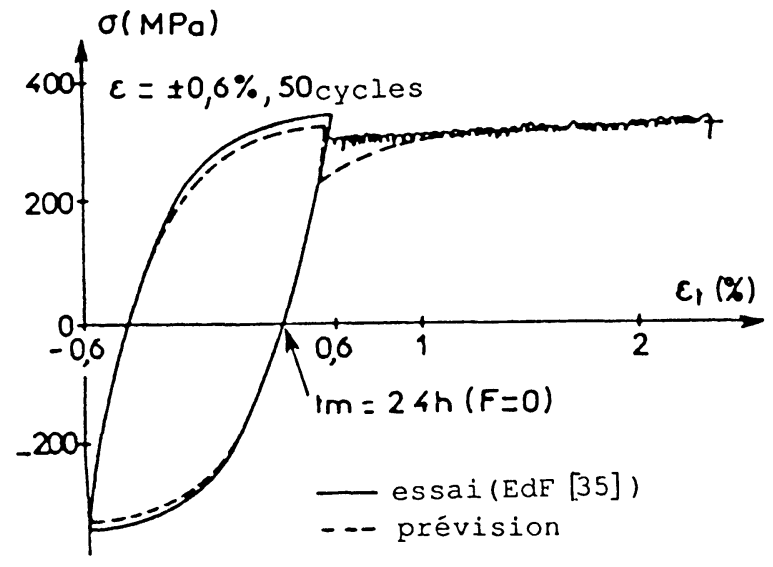

Fig. 18 - Simulation d'un essal de restauration après consolidation cyclique.

[Simulation of a time recovery test after cyclic hardening.]

6. Conclusion.

Le souci constant de décrire le mieux possible le comportement réel de matériaux, conduit à l'introduction, dans les modèles de comportement, de variables supplementaires, pour rendre compte d'effets complexes, tels que les effets de mémoire de 1a deformation plastique ou, à haute température les effets de vieillissement et de restauration.

Ces variables, justifiées sur la base de résultats expérimentaux mais aussi par des considérations plus physiques, augmentent bien sûr le degré de complexité des modèles, mais sont nécessaires pour augmenter la fiabilité des résultats des calculs de structures.

On a fait dans ce papier une synthèse rapide des développements réalisés ces dernières années, que l'on peut résumer de la façon suivante :

- La superposition des modèles d'écrouissage cinématique non linéaire et d'écrouissage isotrope, permet de decrire correctement les effets de base de 1'écrouissage : effet Bauschinger, durcissement

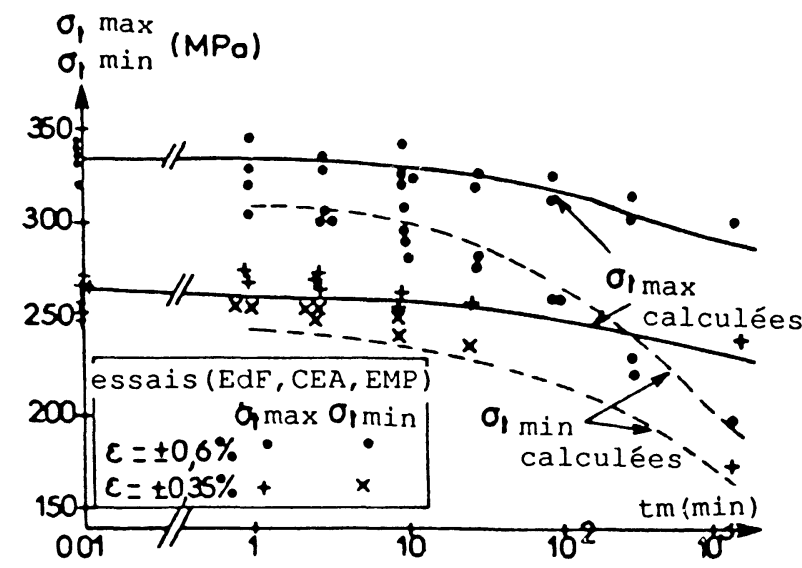

F1g. 19 - Description de l'état stabllisé pour différents temps de maintien.

[Description of the stabilized state for a set of strain controlled-cyclic tests with various hold times in tension.]

et adoucissement cyclique, êtat stabilisé.

- L'introduction d'une variable mémoire, tenant compte de l'influence de la déformation plastique antérieure, permet d'améliorer notablement la description du comportement cyclique pour une large gamme de sollicitations. Elle permet également de décrire, avec un même jeu de coefficients, le comportement du matériau vierge (durcissement cyclique) et celui du matériau précroui (adoucissement cyclique).

- A haute température, 1'introduction d'un terme d'écrouissage par le temps, rend compte des effets de vieillissement et de restauration. De cette façon, une approche unifiée (ne faisant intervenir qu'une seule deformation inélastique) peut rendre compte des nombreux effets d'interaction entre plasticité et fluage, observés notamment sur les aciers inoxydables.

- L'influence de la non-proportionnalité du chargement, qui n'a êtê que brièvement abordée dans ce papier, a également été introduite dans cette classe de modèles.
[1] SIDOROFF, F., Arch. Mech. Poland, Vol. 27 $\mathrm{n}^{\circ}$ 5-6 (1975) p. 807-819.

[2] CHABOCHE, J.L., ROUSSELIER, G., J. of Pressure Vesse1 Technology, Vol. 105 (1983) p. 153.

[3] OYTANA, C., MERMET, A. and DELOBELLE, P., Proc. of the 3rd Int. Conf. on Mechanical Behaviour Material, Combridge (1979).

[4] PRAGER, W., Proc. of the Inst. of Mech. Engineers, London, Vol. 169 (1955) p. 41-50.

[5] ARMSTRONG, P.J. and FREDERICK, C.0., CEGB Report $\mathrm{n}^{\circ} \mathrm{RD} / \mathrm{B} / \mathrm{N} 731$ (1966).

[6] MARQUIS, D., Thèse Paris VI (1979).

[7] CHABOCHE, J.L. Bull de 1'Acad. Polonaise des Sciences. Serce Sc. et Tech. Vol $25 \mathrm{n}^{\circ} 1$ (1977) p. 33-42.

[8] CHABOCHE, J.L., Int. J. of plasticity Vol. 2 $\mathrm{n}^{\circ} 2$ (1986) p. 149-188.
[9] CHABOCHE, J.L., ROUSSEliER, G. Proc. 3rd Int. Seminar "Inelastic Analysis and life prediction in High temperature environment" SMIRT-6, Paris (1981). T.P. ONERA $81-111$.

[10] CHABOCHE, J.L., Proc. of the 2nd Int. Conf. on constitutive Lows for engineering materials. Vol. 1, Tucson (1987). T.P. ONERA 87.

[11] GOODALL, I.W., HALES, R. and WALTERS, D.J., CEGB Report $\mathrm{n}^{\circ} \mathrm{RD} / \mathrm{B} / \mathrm{N} 4916$ (1980).

[12] CHABOCHE, J.L., DANG VAN, K. and CORDIER, G., Proc. of SMIRT 5 Division L, Berlin (1979).

[13] Bollinger, E., CAilletaud, G., CHABOCHe, J.L., DUFAILLY, J., KACZMAREK, H., LIEURADE, H.P., MARQUIS, D. and RIBES, A. Rapport DDSTI, (1982).

[14] NOUAILHAS, D., CAIlletaud, G., POLICELla, H., 
MARQUIS, D., DUFAILLY, J., BOLLINGER, E., LIEURADE, H.P. and RIBES, A. Plasticity Today : Int. Symposium on current trends and results in plasticity, Udine (1983).

[15] OHNO,N., A.S.M.E. J. appl. Mech. 49 (1982) p. 721-727

[16] DAFALIAS, Y.F. and SEYED-RANJDARI, M.S., Proc. 2nd Cairo University Conf. on Mechanical Design and Production (1982).

[17] LAMBA, H.S. and SIDEBOTTOM, O.M., J. of Engng. Mat. and Tech. Vol 100 (1978) p.96.

[18] KANASAWA, K., MILLER, K.J. and BROWN, M.W., Fat. of Engng. Mat. and Structs. Vol 2 p. 217 .

[19] CAILletAuD, G., Mech. of Mater. Vo1 3 (1984) p. 333 .

[20] CHABOCHE, J.L., NOUAIlHAS, D., POLICELla, H., PACOU, D., Rapport ONERA $n^{\circ} 60 / 1765055 \mathrm{R}$ (1986).

[21] Benallal, A., Cailletaud, G., Chaboche, J.l., MARQUIS, D., NOUAILHAS, D., ROUSSET, M., Multiaxial Fatigue Conf., Sheffield (1985) T.P. ONERA $86-13$.

[22] NOUAIlHAS, D., CHABOCHE, J.L., SAVALlE, S., CAILLETAUD, G., Int. J of Plasticity Vol. 1 (1985) p. 317-330.

[23] Benallal, A. MARQuis, D. Proc. 2nd int. Conf. on constitutive Laws for engineering materials Vol. 1 Tucson (1987).

[24] DELOBELle, P., OYTANA, C., J of Nuclear Mat.
139 (1986) p. 204.

[25] NOUAILHAS, D., POLICELLA, H. and KACZMAREK H. Proc. of lst Int. Conf. on constitutive Laws for engineering materials, Tucson (1983) T.P. ONERA 83.07 .

[26] CHABOCHE, J.L., NOUAILHAS, D., Rapport ONERA $n^{\circ}$ 53/1765 RY 003 R (1984).

[27] RABOTNOV, Y.N., North. Holland Publ. Comp. (1969).

[28] LARSSON, B. and STORAKERS, B., J. of Engng Mat. and Tech. Vol. 100 (1978) p. 395-401.

[29] CHO, U.W. and FINDLEY, W.N., ASME J. of Applied Mechanics Vol 48 (1987) p. 785.

[30] PUGH, C.E., Proc. of 3rd Int. Seminar on inelastic analysis and life prediction in High temperature environment, Paris (1981).

[31] STrRUDEL, J.L., Ecole d'été Yravals (1979) Ed. de Physique p. 199.

[32] SCHMIDT, C.G. and MILLER A.K., Res. Mechanica Vol. 3 (1981) p. 109-129.

[33] NOUAILHAS, D., Proc. of 2nd Int. Conf. on constitutive Laws for engineering materials Vol. 1, Tucson (1987). T.P. ONERA 87-01.

[34] CAILlETAUD, G. et CONTESTI, E., Rapport EMP $n^{\circ} 84 / 2 / 43 / 00 / 0$ (1985).

[35] CROUZET, D., Rapport EdF D $592 \mathrm{MAT} / \mathrm{T} 43$. 